\title{
Link Stability Based On Qos Aware On - Demand Routing In Mobile Ad Hoc Networks
}

\author{
Prof.Rekha Patil ${ }^{1}$, Megha Rani.R ${ }^{2}$, Prof.Rajkumar Bainoor ${ }^{3}$ \\ ${ }^{1,2}$ (Department of Computer Science and Engineering, PDA College of Engineering, Gulbarga, India) \\ ${ }^{3}$ (Department of $E \& C E, P D A$ College of Engineering, Gulbarga, India )
}

\begin{abstract}
MANET is a autonomous network where communicating entities can communicate without the aid of any downlink power controller called base stations. The wide deployment of MANET, demand for providing different quality of service $(Q o S)$ in the network has increased much. QoS routing strategy focused on routing strategy for different QoS in this dynamic network. Hence the task of QoS routing is to optimize the network resource utilization while satisfying the application requirements. Therefore there is no centralized control over links. Further the link quality varies due to mobility of nodes. Existing routing protocols like Ad hoc on demand routing protocol (AODV) or other quality of service routing have a scope of not making the changes in link quality once the path is established and cost matrix is set to zero and link quality is not taken in to consideration to select the stable paths. Therefore the performance of such system degrades with high mobility. Hence in this work we emphasis on incorporating link quality estimation based on mobility prediction of nodes incorporate same in the routing decision further new routes are accumulated and the primary transmission path is changed in case of a better route interms of link quality is obtained. In this paper we mingle the idea of link stability and cost matrix, power consumption to uncover better path interms of both stability and cost along with QoS support.
\end{abstract}

Keywords: MANET, Performance metrics, Quality of Service, Routing

\section{Introduction}

A mobile ad hoc network is envisaged as a collection of mobile nodes with no fixed infrastructure and with no central authority. Extensive use of portable mobile devices and the increasing demand of connectivity among the devices have made mobile ad hoc network as one of the flourishing frontier of wireless research. Ad hoc network is a network in which each node is willing to forward data for other nodes and the determination of which nodes forward data is made dynamically based on the network connectivity [1]. Variety of applications exist like personal area networking, disaster management, relief and rescue operation, military, business and other scientific applications. Mobile ad hoc network is a self configured and self maintained network with no centralized authority

Every node in the MANET acts as both a host and a router and must perform some network function. With the development of the MANET, more importance is given to QoS routing strategy. QoS routing strategy [3] focused on routing strategy for different QoS in this dynamic network. Variable link conditions are intrinsic characteristics in most Mobile ad hoc networks. Rerouting among mobile nodes causes network topology in[2] to change dynamically. MANET nodes move in unpredictable manner links between nodes are set up to carry out the communication between the nodes once they become aware of each other. Several QoS routing protocol is being proposed over the years. This claims to provide optimum routing and packet delivery solution to MANET. Most of these QoS techniques are node centric which means the time of processing route request decides if it can provide sufficient QoS to path before forwarding route request. But variations in link quality should incorporate quality of all links through which data transmission is possible. There are major factors like mobility, cost, power and energy efficiency need to be considered to assure better network performance. Especially while assuring QoS in MANET environment nodes should not die due to power constraints or the links should not expire due to mobility in the middle of the transmission. So our target is to choose a more stable path considering higher link stability and less cost along with power variation. Considering these parameters we present a routing algorithm which performs better in terms of cost and link quality. Simulation justifies our claim in the latter section.

\section{Related Work}

An Ad hoc network is a network in which each node is willing to forward data for other nodes and the determination of which nodes forward data is made dynamically based on the network connectivity an important property of ad hoc network is the multi-hop capability and applications of mobile ad hoc network are discussed [4] towards efficient delivery of services. 
A good number of routing protocols [5] already been proposed for mobile ad hoc network. Proposed routing protocols are often subdivided in two categories: Proactive (table driven) and reactive (on demand) routing protocol. The on demand routing protocol AODV (Ad hoc on demand routing protocol) is a novel algorithm for the operation of Ad hoc networks this protocol is an excellent choice for ad hoc network establishment is proposed in[6][7] the phases involved in AODV are path discovery, route table management, path maintenance,(RREQ,RREP,RERR) are the control messages used for establishing paths in the MANET. Periodic hello messages can be used to ensure symmetric links as well as to detect link failures. [8] When a node sends unsolicited RREP message to each of the source sending the traffic through the failed link to their neighbors.

The Cross Layer violates the OSI layered architecture but keep the impact of design violations as small as possible. Such violation of a layered architecture is cross layer design interfaces are created between the layers in the CLD [9-11]. The most suitable design for communication in wireless networks is Cross Layer Design (CLD). There are various cross layer design methods available. A simple and effective design of the cross layer architecture has been chosen to improve the performance of lot of services in ad hoc networks. In our paper, we are using upward information flow. A higher-layer protocol that require some information from the lower layer(s) at runtime results in creation of a new interface from the lower layers to the higher layer.

In this paper the author discusses various QoS aware routing protocols and parameters [12].QoS aware routing is of great importance in MANETs since it can improve the performance and allow critical information to flow even under difficult conditions [15] there is necessity to measure link stability in order to provide QoS while selecting route. One of the main challenges in providing QoS in MANETs is mobility of the nodes, which makes connectivity unpredictable. QoS routing in MANET is usually limited by the network breakage due to either node mobility or energy depletion of the mobile nodes. Link duration and link quality are measured [13].

The major problem with Ad hoc network is link quality variations due to mobility [14] of nodes. Link stability, distance, velocity between nodes is discussed in [15][16] link quality is the most promised parameters, since it define the ability of a link and devices to support the density of the traffic for the period of connection. The link state between two neighbors can be affected by many parameters [17] such as distance, power and mobility. Link stability models are discussed in [18] where link stability estimation model is proposed using periodic beacons signals. Different characteristics of link stability are discussed [19].

\section{Proposed Work}

Ad hoc wireless routing protocols typically select the route with the low cost or minimum hop count to the destination. However, routes chosen according to such criteria tend to include longer hop length links with possibly bad signal quality links. So, the routing protocol must be improved by eliminating the usage of routes with bad links.

The main scope of our work is to ensure QoS through link quality in MANET along with ensuring transmission through best path in terms of less power loss. Link quality is one of the challenges in MANET which effects the transmission quality, used to transmit real time traffic. This work can be used to find the stable paths with minimum probability of link failures in the path. Power loss can also be a parameter for representing link stability.

In this proposed work we emphasis on incorporating link quality estimation based on mobility prediction of nodes incorporate same in the (AODV) routing decision to avoid routing through the bad quality links and establish new routes and transmission path is changed a better route in terms of link quality is obtained. A technique is proposed for estimating the link quality between the nodes. Each node finds its position through the GPS (Global Positioning System) i.e., every physical node is equipped with a GPS [20].Where each every node broadcasts $\mathrm{X}, \mathrm{Y}$ to the neighbors through hello packet.

The distance between the nodes is calculated based on positional coordinates $\mathrm{x} 1, \mathrm{x} 2$, $\mathrm{y} 1$ and $\mathrm{y} 2$ for the two nodes which are in the transmission range $\mathrm{r}$ and considering velocity $\mathrm{v} 1, \mathrm{v} 2$ and direction $\theta 1$ and $\theta 2$ respectively. The distance is given by:

$$
\mathrm{D}=\sqrt{(\mathrm{x} 2-\mathrm{x} 1) 2+(\mathrm{y} 2-\mathrm{y} 1) 2}
$$

Where $\mathrm{D}$ is the distance between two nodes, $\mathrm{t}$ is the time period

Link stability between the nodes is calculated by:

$$
\mathrm{L}_{\mathrm{st}}=\text { Range/Distance }
$$

Finally the link stability is calculated in terms of cost between the nodes. Cost is the major factor based on which the path is considered as stable.

$$
\text { Cost }=\mathrm{L}_{\mathrm{st}}+1 / \text { power }
$$

This positional data is also appended in route request and send to destination. The path with minimum cost and power variation is selected as stable path. 


\section{System Design And Implementation}

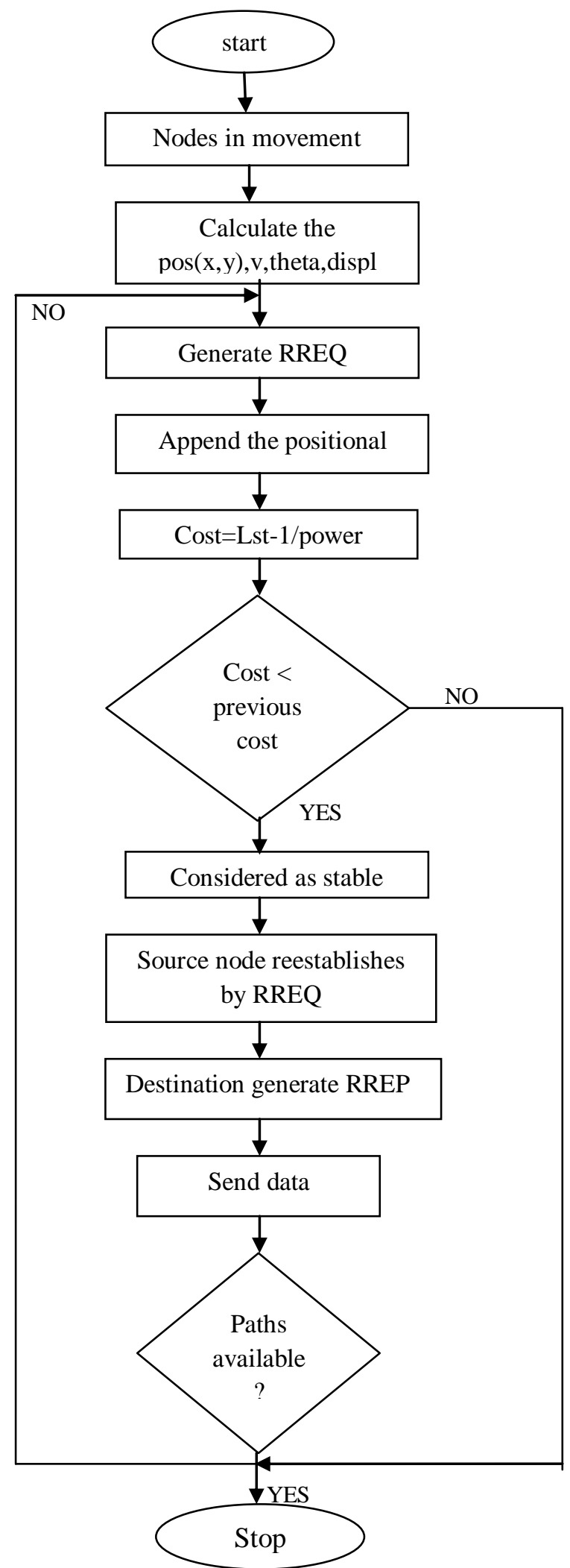

Figure 1: The flow chart of proposed work.

This paper has been implemented to know whether the paths used for routing the packets by nodes are stable or not?

AODV routing protocol is used for routing the packets in the network. The fig (1) illustrates that when nodes are in mobile, using GPS the positional coordinates $\mathrm{x}, \mathrm{y}, \mathrm{v}$ (velocity), theta,displacement are calculated. 
Link Stability Based on QoS Aware on - Demand Routing in Mobile Ad hoc Networks

These parameters are appended in RREQ and based on cost the path is selected for routing. If the cost is high than the previous path then that path is rejected. The path with the low cost is selected as a stable for routing.

\section{Simulation}

To study the performance of our proposed algorithm simulation was conducted using OMNeT++ [21] which supports complete physical, data link and MAC layer models for simulating wireless ad hoc networks. OMNeT++is a freely distributed, object oriented, modular, discrete event simulator written in c++. It is designed for general- purpose discrete-event simulation, and provides model libraries for communication protocols and network systems. We simulated network of mobile nodes placed randomly in an area of $300 * 400$ square meters, with mobile nodes ranging from 10 to 50.A source and a destination is selected randomly. Free space propogation model is assumed as the channel model. Each node is assumed to have constant transmission range of 250 meters. Mobility pattern of the mobile nodes is generated using Random way point model. A mobile node selects another node in the network and constantly moves towards it at a given velocity. Once it reaches there, it waits for some pause time and selects another node and again starts moving. By observing the performance of the network under mobility we can test the stability of the design in real time scenario. Speed of mobile node is assigned a value between 1 to 4 meters/sec. Initially all the mobile nodes would be given some initial energy (10000). As the packets are to be transmitted through the nodes, they would lose some energy. It is assumed from previous research [22](as the size of each packet is constant), that the radio interface, when powered on, consumes $0.3 \mathrm{~mW}$ while actually while receiving a packet and $0.9 \mathrm{~mW}$ while transmitting a packet. As the communication in MANET is normally a cross layered approach, upward information flow model is very efficient in designing and modeling the network. The performance parameters studied for simulation study are throughput, latency, packet delivery ratio and control overhead by varying the pause time, simulation time and load.

\subsection{Results}

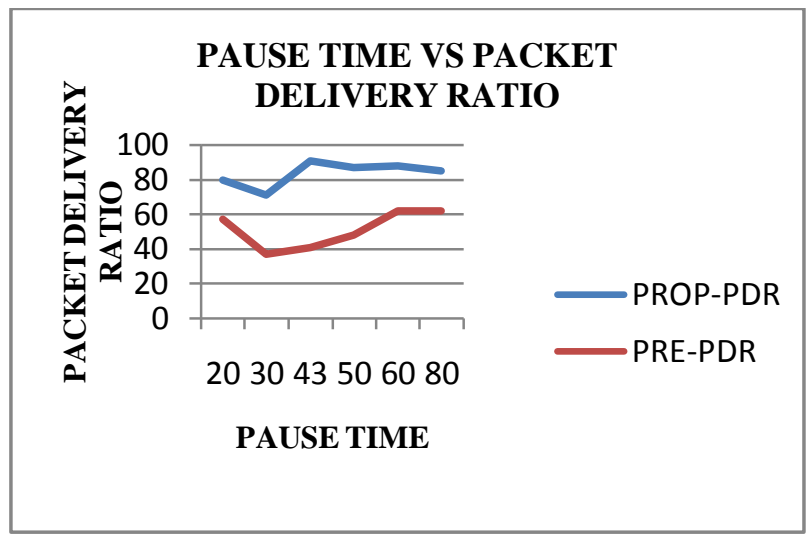

Figure 2: Packet delivery ratio Vs Pause time

Fig (2) shows packet delivery ratio achieved by proposed protocol. Improved packet delivery ratio is achieved due to the fact that the proposed protocol is to choose stable paths. Increasing the pause time effects the proposed system. Packet delivery ratio does not effect with increasing pause time.

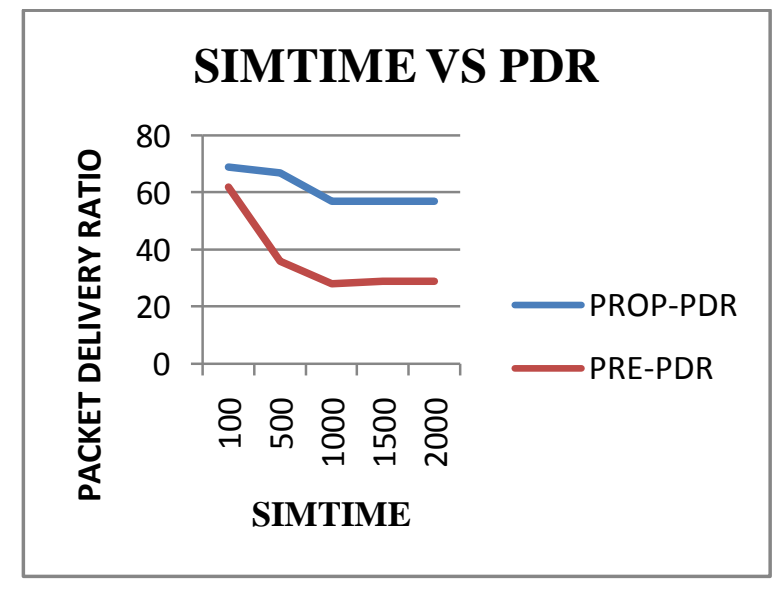

Figure 3: Packet delivery ratio vs Simulation time 
Fig(3) shows the packet delivery ratio is better for proposed system than the existing system .As the simulation time is increased the packet delivery ratio is not effected.

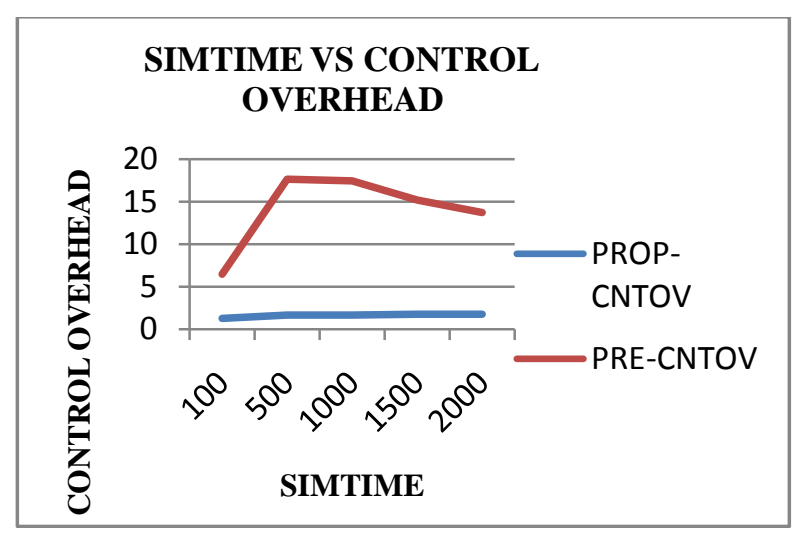

Figure 4: Control overhead Vs Simulation time

The control overhead is always almost constant in proposed system. Control overhead is less when compared to the present system. The system performs better when control overhead is increased.

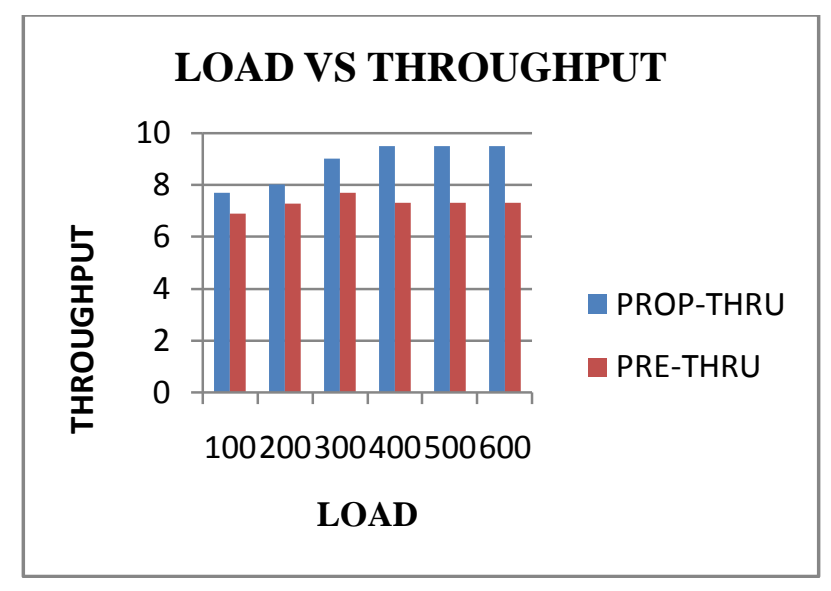

Figure 5: Throughput Vs Load

The throughput obtained is better for proposed as compared to present. As the rate of transmission of packets (load) increases the throughput increases for our proposed system. The cross layer approach enables to transmit at a rate to obtain high throughput.

\subsection{Performance Metrics}

Throughput: Network throughput is the average rate of successful bits delivery over a communication channel. Channel utilization per second is known as throughput. The throughput is usually measured in bits per second (bits/s or bps).

Control overhead: It is defined as number of control packets transmitted for every data packet delivered.

Packet delivery ratio: It is defined by a factor of number of packets received by number of packets transmitted. Pause time: It is the time period between two subsequent movements in the mobility model of a node.

Packet rate (load): The number of packets per second going in or coming out of a given server.

Latency: It is the amount of time a message takes to traverse a system.

\section{CONCLUSION And FUTURE WORK}

We present a new technique for simulation study to ensure several challenges in continuous and quality transmission one of the major causes for performance degradation like packet drops are due to link losses and link quality variations. Several link aware routings are also proposed which primarily determines the link quality on power or signal to noise ratio. But experimental studies have shown that one of the major causes for link failure is mobility of nodes, which directly effects the power losses in the transmission. 
Therefore in this work we propose link quality aware routing where link quality is measured using prediction of node positions through the past history of nodes mobility. We also propose a unique mechanism of accumulating new path as and when a path with better link quality is available. The cost matrix is calculated based on link stability between nodes and power is also calculated. It has some limitation random mobility of nodes with very high speed produces improper link quality measurements, multipath fading and wide channel noise affects the link quality. In MANET link quality is one of the challenges which effects the transmission quality, which is used to transmit real time traffic like multimedia etc.

A discrete-event $\mathrm{C}++$ based simulator OMNeT++ can efficiently simulate proposed work. From the simulation results we can conclude that the throughput and packet delivery ratio are significantly improved in the proposed work. Control overhead is reduced to ensure better performance. There is however slight increase in latency which is attributed by more packet delivery and layer updation.

The system can be further included by incorporating more matrices for link quality assessment like channel noise, channel capacity, bit error rate, packet collisions and so on and by determining the best path using statistical classifier Baysen or nearest neighbor classifier.

\section{References}

[1] Okeke, S. S. N, and Nwabueze, C.A., Mobile Ad hoc Network (MANET) architecture and implementation Analysis, Natural and Applied Sciences Journal Vol.11 No.1.

[2] M-Mamum-Or-Rashid and Choong Seon Hong, LSLP: Link stability and lifetime prediction Based QoS Aware Routing for MANET, Department of Computer Engineering.

[3] Yihai Zhang, Quality of Service for Ad hoc on-demand Distance Vector Routing, 1996.

[4] Jiazi YI, Polytech Nantes, A Survey on the Applications of MANET, Feb 2008.

[5] C E perkins and P.Bhagwat, Highly Dynamic Destination Sequenced, distance vector routing(DSDV) for mobile computers, In Proc. ACM SIGCOMM, pp.234-244, 1994.

[6] Charles E perkins and Elizabeth M Royer, Ad hoc on-demand Distance Vector routing.

[7] Charles E Perkins and Elizabeth M. Royer, Samir R. Das and Mahesh K., Performance Comparision of Two on-demand Routing Protocols for Ad hoc Networks, IEEE personal communications, Feb 2001, pp-16-28.

[8] Laura Marie Fenny, A Energy Consumption Model for Performance Analysis of Routing Protocols for Mobile Ad hoc Networks, Mobile Networks and applications, Vol 6, pp-239-249,2001.

[9] Philip Hurni, Cross-Layer Design in Wireless Networks, April 2008.

[10] Jeffery Mehlman,” Cross-Layer Design : A Case for Standardization”,Jmehlman@ stanford.edu.

[11] Vineet Srivastava, Mehul Otani, Cross-Layer Design: A survey and the Road Ahead, IEEE communications Magazine, December 2005.

[12] Shan Gong, Quality of Service Aware Routing Protocols for Mobile Ad hoc Networks, 2006.

[13] Shuchita Upadhayaya, Charu Gandhi, QoS Routing using Link and Node Stability in Mobile Ad hoc Networks, Journal of Theoritical and Applied Information Technology 2009.

[14] Fan Bai and Ahmed Helmy, A Survey of Mobility models in Wireless Ad hoc Networks.

[15] S. Muthuramlingam and R. Rajaram, A Transmission Range Based Clustering Algorithm for Topology Control MANET, International Journal on applications of graph theory in Wireless ad hoc networks Vol2,No.3,September 2010.

[16] Aarthi Agarwal, Samir R Das, Dead Reckoning in Mobile Ad hoc Networks, Computer Science and Engineering Department.

[17] Benamar KADRI, Djilali MOUSSAOUI, Mohammed FEHAM, Link Quality Based Ant Routing Algorithm for MANETs(LQARA), 2011 PGNeT.

[18] Min-Gu lee and Suggu lee, A link Stability Model and Stable Routing for Mobile Adhoc Networks, Electrical and Computer Engineering Division.

[19] Michael Gerharz, Christian dewaal, Matthias Frank peter Martini, Link Stability in Mobile Wireless Ad hoc Networks, Institute of Computer Science.

[20] Sr dan Capkun, Maher Hamdi, Jean-pierre, GPS-Free Positioning in Mobile Ad hoc Networks, 2001.

[21] Andras Verga, OMNeT++, www.omnetpp.org.

[22] L.M. Fenny, Energy Consumption Model for Performance analysis of Routing Protocols for Mobile Ad hoc Networks , ACM J. of Mobile Networks and Applications, Vol.6 issue 3, June 2001. 